\title{
Primal-dual interior point method for multicommodity network flows with side constraints and comparison with alternative methods
}

\section{J. Castro, N. Nabona}

Statistics and Operations Research Dept., Univ. Politècnica de Catalunya Pau Gargallo 5, 08071 Barcelona, Spain.

Tel: 34-3-4017335. Fax: 34-3-4017040. e-mail: jcastrop@eio.upc.es

\begin{abstract}
This document presents a primal-dual interior point algorithm for the solution of large multicommodity network flow problems with or without side constraints. The method exploits the structure of the problem and uses a preconditioned conjugate gradient solver. The algorithm has been implemented for the case of pure multicommodity problems (without side constraints), and some computational results are presented, comparing the performance of the code developed with alternative ones.
\end{abstract}

\section{Keywords}

Computational Benchmarks, Interior Point Methods, Linear Programming, Multicommodity Network Flows, Primal-Dual Algorithm, Side Constraints

\section{INTRODUCTION}

Multicommodity network flows (Kennington and Helgasson (1980)) are used as a modelling tool in many applications in routing, telecommunication networks, allocation and transportation problems and in electrical power systems. It is thus important to have efficient tools to optimize this kind of problems. Interior point methods have recently gained wide recognition as an optimization procedure for applications with general linear constraints and both their general primal-dual and dual-affine-scaling formulations have been extended to the linear multicommodity network flow problem (Choi and Goldfarb (1990), Kamath et al. (1993)).

In the work described here the primal-dual interior point algorithm has been specialized for solving multicommodity network flow problems, considering additional side constraints. The method is heavily based on the use of a preconditioned conjugate gradient algorithm for solving repeatedly a part of the systems of the type $A S A^{t} d y=\bar{b}$, $A S A^{t}$ being symmetric and positive definite. Two specialized multicommodity network 
flow codes (Kennington (1979), Castro and Nabona (1995)), both based on the primal partitioning algorithm (Kennington and Helgasson (1980)), have been run on the same test problems solved with the interior point code in order to compare the performance of the interior point solution with that of the specialized network codes. The performance of the multicommodity primal-dual interior point code developed is also compared with that of a general primal-dual interior point code (Vanderbei (1993)) so that it is possible to appreciate the computational advantages of using multicommodity specialization within the interior point scheme. Randomly generated multicommodity test problems of sizes ranging from 100 to 10000 arcs and numbers of commodities ranging from 1 to 200 were solved and their results are reported.

\section{OUTLINE OF THE PRIMAL-DUAL INTERIOR POINT FOR UPPER-BOUNDED LINEAR PROGRAMMING}

Let us consider the following minimization problem with upper bounds in some variables

$$
\begin{aligned}
\min & c^{t} x \\
\text { subj. to } & A x=b \\
& \underline{0} \leq x_{u} \leq \bar{x}_{u} \\
& \underline{0} \leq x_{l}
\end{aligned}
$$

where $x_{u} \in \mathbb{R}^{n_{u}}, x_{l} \in \mathbb{R}^{n_{l}}, x=\left(x_{u}^{t}, x_{l}^{t}\right)^{t}, x \in \mathbb{R}^{n}$ (thus $n=n_{u}+n_{l}$ ), $c \in \mathbb{R}^{n}, b \in \mathbb{R}^{m}$ and $A \in \mathbb{R}^{m \times n}$. Considering an appropriate partitioning of $c$ and $A$, equations (1) and (2) can be rewritten as:

$$
\begin{gathered}
c_{u}^{t} x_{u}+c_{l}^{t} x_{l} \\
A_{u} x_{u}+A_{l} x_{l}=b
\end{gathered}
$$

where $c_{u} \in \mathbb{R}^{n_{u}}, c_{l} \in \mathbb{R}^{n_{l}}, A_{u} \in \mathbb{R}^{m \times n_{u}}$ and $A_{l} \in \mathbb{R}^{m \times n_{l}}$. The dual of the minimization problem stated can be cast as:

$$
\begin{aligned}
\max & b^{t} y-\bar{x}_{u}^{t} w \\
\text { subj. to } & A_{u}^{t} y+z_{u}-w=c_{u} \\
& A_{l}^{t} y+z_{l} \quad=c_{l} \\
& z=\left(z_{u}^{t}, z_{l}^{t}\right)^{t} \geq 0 \quad w \geq 0
\end{aligned}
$$

where $z_{u} \in \mathbb{R}^{n_{u}}, z_{l} \in \mathbb{R}^{n_{l}}$ (thus $z \in \mathbb{R}^{n}$ ) and $w \in \mathbb{R}^{n_{t u}}$.

Considering a logarithmic barrier function ( $\mu$ being its penalty term) for the nonnegativity constraints of the variables, and adding slacks $f \in \mathbb{R}^{n_{u}}$ for the upper bounds $\left(x_{u}+f=\bar{x}_{u}\right)$, the Kuhn-Tucker optimality conditions for both the dual and the primal can be written as:

$$
\begin{array}{r}
b_{1_{l}} \equiv \mu e_{n_{l}}-X_{l} Z_{l} e_{n_{l}}=0 \\
b_{1_{u}} \equiv \mu e_{n_{u}}-X_{u} Z_{u} e_{n_{u}}=0 \\
b_{2} \equiv \mu e_{n_{u}}-F W e_{n_{u}}=0 \\
b_{3} \equiv b-\left(A_{u} x_{u}+A_{l} x_{l}\right)=0 \\
b_{4_{l}} \equiv c_{l}-\left(A_{l}^{t} y+z_{l}\right)=0 \\
b_{4_{u}} \equiv c_{u}-\left(A_{u}^{t} y+z_{u}-w\right)=0
\end{array}
$$


$e_{l}$ being the $l$-dimensional vector of 1 's, and where matrices $X_{u}, X_{l}, Z_{l}, Z_{u}, F$ and $W$ are diagonal and defined as $M \in \mathbb{R}^{l \times l}=\operatorname{diag}\left(m_{1}, \ldots, m_{l}\right)$. It is clear than when $n_{u}=\mathbf{0}$ $\left(n=n_{l}\right)$ only equations $(7,10$ and 11$)$ hold, thus having the optimality conditions of the standard primal-dual algorithm with no upper bounds.

When using Newton's method to find a point satisfying (7-12), linear systems of the type $J_{i} d_{i}=-f_{i}$ must be solved at each iteration $i$. These solutions amount to finding $d y$ and then computing $d x, d w, d z_{u}, d z_{l}$, in:

$$
\begin{aligned}
\left(A S A^{t}\right) d y & =b_{3}+A S r \\
d x & =S\left(A^{t} d y-r\right) \\
d w & =F^{-1}\left(b_{2}+W d x_{u}\right) \\
d z_{u} & =b_{4_{u}}+d w-A_{u}^{t} d y \\
d z_{l} & =b_{4_{l}}-A_{l}^{t} d y
\end{aligned}
$$

where

and

$$
\begin{gathered}
r=\left(r_{u}^{t}, r_{l}^{t}\right)^{t} \quad r \in \mathbb{R}^{n} \quad r_{u} \in \mathbb{R}^{n_{u}} \quad r_{l} \in \mathbb{R}^{n_{l}} \\
r_{u}=F^{-1} b_{2}+b_{4_{u}}-X_{u}^{-1} b_{1_{u}} \quad r_{l}=b_{4_{l}}-X_{l}^{-1} b_{1_{l}}
\end{gathered}
$$

$$
\begin{gathered}
S=\left(\begin{array}{cc}
S_{u} & \mathbf{0} \\
\mathbf{0} & S_{l}
\end{array}\right) \quad S \in \mathbb{R}^{n \times n}, \quad S_{u} \in \mathbb{R}^{n_{u} \times n_{u}}, \quad S_{l} \in \mathbb{R}^{n_{l} \times n_{l} u} \\
S_{u}=F X_{u}\left(Z_{u} F+X_{u} W\right)^{-1} \quad S_{l}=Z_{l}^{-1} X_{l}
\end{gathered}
$$

(where $S_{u}$ and $S_{l}$ can be directly computed, since they are made of products and sums of diagonal matrices). A justification of this process can be found in Castro (1995a).

It is quite clear that the main computational burden in solving system (7-12) is the repeated solution of the linear system (13).

\section{FORMULATION OF THE LINEAR MULTICOMMODITY NETWORK FLOWS WITH SIDE CONSTRAINTS}

The multicommodity network flow problem corresponds to the minimization problem (1-4). Let us consider a network with $m^{*}$ nodes, $n^{*}$ arcs (where the last one is a rooted arc added to avoid the singularity of the network matrix $\left.A^{*} \in \mathbb{R}^{m^{*} \times n^{*}}\right)$ and $k$ commodities. Adding slacks to the mutual capacity constraints $\left(s_{m c} \in \mathbb{R}^{n^{*}}\right)$ and the side constraints $\left(s_{s c} \in \mathbb{R}^{t}, t \geq 0\right)$, and denoting by $x_{i}=\left(x_{i_{a}}^{t} x_{i_{r}}\right)^{t} \in \mathbb{R}^{n^{*}} i=1, \ldots, k$ the flows for each commodity $\left(x_{i_{r}} \in \mathbb{R}\right.$ being the rooted arc and $x_{i_{a}} \in \mathbb{R}^{n^{*}-1}$ the remaining ones, for commodity $i$ ) with capacities $\bar{x}_{i} \in \mathbb{R}^{n^{*}-1}$ (thus considering the rooted arcs as uncapacitated ones), by $b_{m c} \in \mathbb{R}^{n^{*}}$ the mutual capacities (for the rooted arcs an arbitrary mutual capacity can be considered), by $b_{i} \in \mathbb{R}^{m^{*}} i=1, \ldots, k$ the node supplies/demands of each commodity $i$, by $T_{i} \in \mathbb{R}^{t \times n^{*}}$ the matrices defining the side constraints structure, and by $\bar{b}_{s c}, \underline{b}_{s c} \in \mathbb{R}^{t}$ the upper and lower bounds of the side constraints, then the multicommodity network problem can be stated as: 


$$
\min \sum_{i=1}^{k} c_{i}^{t} x_{i}
$$

subj. to

\begin{tabular}{|c|c|c|c|c|c|c|c|c|}
\hline$A^{*}$ & 0 & $\ldots$ & 0 & 0 & 0 & $x_{1}$ & & $b_{1}$ \\
\hline 0 & $A^{*}$ & $\ldots$ & 0 & 0 & 0 & $x_{2}$ & & $b_{2}$ \\
\hline$\vdots$ & $\vdots$ & $\ddots$ & $\vdots$ & $\vdots$ & $\vdots$ & $\vdots$ & - & $\vdots$ \\
\hline 0 & $\mathbf{0}$ & $\ldots$ & $A^{*}$ & 0 & 0 & $x_{k}$ & & $b_{k}$ \\
\hline $\mathbb{1}$ & $\mathbb{1}$ & $\ldots$ & $\mathbb{1}$ & 1 & 0 & $s_{m c}$ & & $b_{m c}$ \\
\hline$T_{1}$ & $T_{2}$ & $\ldots$ & $T_{k}$ & 0 & $\mathbb{1}$ & $s_{s c}$ & & $\bar{b}_{g c}$ \\
\hline
\end{tabular}

In this case the total number of variables and constraints is given by $n=(k+1) n^{*}+t$ and $m=$ $k m^{*}+n^{*}+t$, and the partitioning of the variables $x=\left(x_{u}^{t}, x_{l}^{t}\right)^{t}$ is $x_{u}^{t}=\left(x_{1_{a}}^{t}, \ldots, x_{k_{a}}^{t}, s_{m c}^{t}, s_{s c}^{t}\right)$ and $x_{l}^{t}=\left(x_{1_{r}}, \ldots, x_{k_{r}}\right)$.

In the multicommodity problem, matrix $S$ defined in (19) can be partitioned as:

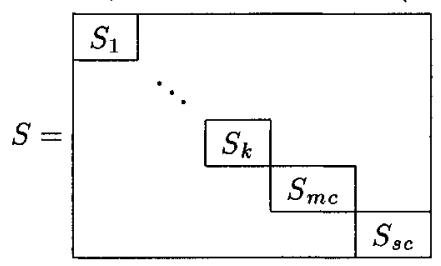

Applying equations (13-17) to the multicommodity problem, one can take advantage of the special structure of matrix $A$, especially when solving $\left(A S A^{t}\right) d y=b_{3}+A S r$. In this case, and considering (21) and (25), the structure of matrix $A S A^{t}$ is as follows:

$\begin{aligned} A S A^{t} & =\begin{array}{|c|c|c|c|c|c|}\hline A^{*} S_{1} A^{*^{t}} & \mathbf{0} & \ldots & \mathbf{0} & A^{*} S_{1} & A^{*} S_{1} T_{1}^{t} \\ \hline 0 & A^{*} S_{2} A^{*^{t}} & \ldots & \mathbf{0} & A^{*} S_{2} & A^{*} S_{2} T_{2}^{t} \\ \hline \vdots & \vdots & \ddots & \vdots & \vdots & \vdots \\ \hline \mathbf{0} & \mathbf{0} & \ldots & A^{*} S_{k} A^{*^{t}} & A^{*} S_{k} & A^{*} S_{k} T_{k}^{t} \\ \hline S_{1} A^{*^{t}} & S_{2} A^{*^{t}} & \ldots & S_{k} A^{*^{t}} & \sum_{i=1}^{k} S_{i}+S_{m c} & \sum_{i=1}^{k} S_{i} T_{i}^{t} \\ \hline T_{1} S_{1} A^{*^{t}} & T_{2} S_{2} A^{*^{t}} & \ldots & T_{k} S_{k} A^{*^{t}} & \sum_{i=1}^{k} T_{i} S_{i} & S_{s c}+\sum_{i=1}^{k} T_{i} S_{i} T_{i}^{t} \\ \hline B & C_{1} & C_{2} \\ \hline C_{1}^{t} & D_{1} & D_{2} \\ \hline C_{2}^{t} & D_{3} & D_{4} \\ \hline\end{array}\end{aligned}$




\section{NUMERICAL SOLUTION SCHEME}

Because of the structure of $A^{*} S_{i} A^{*^{t}}$ and that of $A^{*} S_{i}$, when a solution for system (13) is attempted directly using the Cholesky decomposition, submatrix $D_{1}$ become completely dense. Since the dimension of $D_{1}$ is $n^{*}$ this would mean having to store and process $n^{*}\left(n^{*}+1\right) / 2$ values. For large networks this amount of memory can become prohibitive. This is stated in Choi and Goldfarb (1990), but no procedure is given there to circumvent this difficulty. The algorithm developed considers the solution of the linear system (13) $\left(A S A^{t} d y=\bar{b}\right)$ taking into account the partition indicated in (26); thus the system to be solved can be written as:

\begin{tabular}{|c|c|}
\hline$B$ & $C$ \\
\hline$C^{t}$ & $D$ \\
\hline
\end{tabular} \begin{tabular}{|l|}
\hline$d y_{1}$ \\
\hline$d y_{2}$ \\
\hline
\end{tabular}$=$\begin{tabular}{|l|}
\hline $\bar{b}_{1}$ \\
\hline $\bar{b}_{2}$ \\
\hline
\end{tabular}

whose solution is directly obtained by block multiplication:

$$
\begin{aligned}
\left(D-C^{t} B^{-1} C\right) d y_{2} & =\left(\bar{b}_{2}-C^{t} B^{-1} \bar{b}_{1}\right) \\
B d y_{1} & =\left(\bar{b}_{1}-C d y_{2}\right)
\end{aligned}
$$

$B$ is made of $k$ diagonal blocks $A^{*} S_{i} A^{*^{t}}$. Each block has the same very sparse topological structure of nonzero elements. If $\mathcal{A}$ is the set of arcs of the network, and $\mathcal{I}_{v}$ the set of incident arcs to node $v, A^{*} S_{i} A^{*^{t}}$ can be computed as follows:

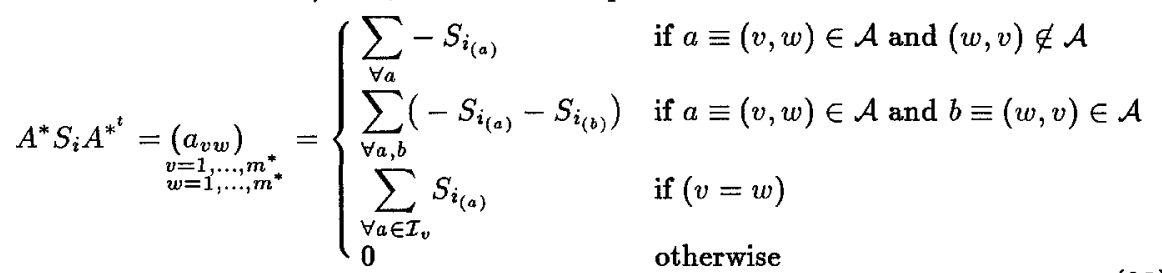

and any solution having $B$ as the system matrix can be decomposed in $k$ systems of equations (thus the process could be parallelized). The minimum order degree algorithm was used to reorder the nodes of the network to avoid fill-in when making the Cholesky decomposition of the $k$ blocks of $B$. Neither the calculation of $B^{-1} \bar{b}_{1}$ nor the solution of $B d y_{1}=\left(\bar{b}_{1}-C d y_{2}\right)$ involves too much work. However, when computing $d y_{2}$, matrix $\widehat{D}=$ $D-C^{t} B^{-1} C$ should be formed, which would mean solving $n^{*}+t$ systems of equations to obtain $B^{-1} C$ and, afterwards, a Cholesky decomposition of $\widehat{D}$.

A better choice is to use a preconditioned conjugate gradient (PCG) algorithm to obtain $d y_{2}$, and then to compute $d y_{1}$ directly. In the PCG algorithm the only operation directly made with the system matrix is a product of it by a vector $v$. But we have: $\left(D-C^{t} B^{-1} C\right) v=D v-C^{t} B^{-1} w$, with $w=C v$. Thus one can take advantage of the sparsity of $D$ and $C$, and the fact that the computation of $B^{-1} w$ is numerically efficient.

In order to speed up the PCG algorithm, a positive definite matrix $M$ must be determined such that $M^{-1} \widehat{D}$ becomes less ill-conditioned than $\widehat{D}$, and that the computation of $M z=r$ does not involve too much work. For pure multicommodity network problems (without side constraints) the system matrix is $\widehat{D}_{1}=D_{1}-C_{1}^{t} B^{-1} C_{1}$. In this case, 
and considering a splitting of matrix $\widehat{D}_{1}$ such that $\widehat{D}_{1}=P-Q$, where $P=D_{1}$ and $Q=C_{1}^{t} B^{-1} C_{1}$ (both positive definite), it can be proved (Castro (1995b)) that:

$$
\widehat{D}_{1}^{-1}=\left(\sum_{i=0}^{\infty}\left(P^{-1} Q\right)^{i}\right) P^{-1}
$$

The preconditioner $M^{-1}$ to be used will be an approximation of $\widehat{D}_{1}^{-1}$, and can be obtained by truncating (29) at some term $\phi$ :

$$
M^{-1}=\left(\mathbb{1}+\left(P^{-1} Q\right)+\left(P^{-1} Q\right)^{2}+\ldots+\left(P^{-1} Q\right)^{\phi}\right) P^{-1}
$$

The higher $\phi$ is, the better the preconditioning, and the fewer iterations of the PCG will be required. However, it must be noted that the product of $Q$ by a vector $r$ implies the solution of $B^{-1}\left(C_{1} r\right)$, and this should be performed at each iteration of the PCG algorithm, increasing the execution time considerably. Thus $\phi$ must be chosen in order to balance both objectives: to decrease the PCG iterations and to improve the time per PCG iteration. Various tests have shown than, in general, the best results are obtained with $\phi=1$. In this case $M^{-1}=P^{-1}=D_{1}^{-1}$, which means that $z=M^{-1} r$ can be obtained in $O(n)$ operations (since $D_{1}$ is a diagonal matrix).

\section{COMPUTATIONAL RESULTS}

The multicommodity primal-dual interior point algorithm outlined in the above sections was implemented for the case of problems without side constraints, using the preconditioning previously stated. The code was written in ANSI-C, and to test its performance four types of problems, obtained from different network generators, were used: Rmfgen, Grid-on-torus, Gridgraph and Gridgen (Dimacs (1991)). These generators do not consider the case of multicommodity flows, and the output networks had to be converted to a multicommodity one. The conversion algorithm is described in Castro (1995b). Five particular instances were created with each of these generators. The first two are problems with few commodities and medium-sized networks, whereas the last three correspond to small-sized networks with many commodities. Each problem will be denoted by $\mathrm{L}_{j}^{i}, i=1, \ldots, 4, j=1, \ldots, 4, i$ denoting the generator employed ( 1 for Rmfgen, 2 for Grid-on-torus, 3 for Gridgraph and 4 for Gridgen). Table 1 presents the characteristics of each problem, showing for each test problem the number of commodities, nodes and arcs of the network, and the total number of constraints and variables of the linear program to be solved (columns Rows $A$ and Columns $A$ ).

The interior point multicommodity code developed (denoted by IPM) was compared with MINOS 5.3 (Murtagh and Saunders (1983)), a general-purpose package, PPRN (Castro and Nabona (1995)) and MCNF85 (Kennington (1979)), two specialized multicommodity network flow codes, and LoQo (Vanderbei (1993)), a state-of-the-art primaldual interior point code. Table 2 shows the CPU seconds required by each code. The fastest execution for each test is marked with an asterisk (*). All runs were carried out on a SunSparc $10 / 41$ (one CPU), with a $40 \mathrm{MHz}$ clock, $\approx 100 \mathrm{Mips}$ and $\approx 20 \mathrm{Mflops} \mathrm{CPU}$, and $64 \mathrm{Mbytes}$ of main memory. From Table 2 it can be concluded that the performance of IPM increases with the size of the problem, this code thus being a good choice 
Table 1 Linear test problems

\begin{tabular}{llllll}
\hline Test & Commodities & Nodes & Arcs & Rows A & Columns A \\
\hline $\mathrm{L}_{1}^{1)}$ & 8 & 2048 & 9472 & 25856 & 85248 \\
$\mathrm{~L}_{2}^{1)}$ & 16 & 2048 & 9472 & 42240 & 161024 \\
$\mathrm{~L}_{3}^{1)}$ & 50 & 128 & 496 & 6896 & 25296 \\
$\mathrm{~L}_{4}^{1)}$ & 150 & 128 & 496 & 19696 & 74896 \\
$\mathrm{~L}_{5}^{1)}$ & 200 & 128 & 496 & 26096 & 99696 \\
$\mathrm{~L}_{1}^{2)}$ & 8 & 1500 & 9000 & 21000 & 81000 \\
$\mathrm{~L}_{2}^{2)}$ & 16 & 1500 & 9000 & 33000 & 153000 \\
$\mathrm{~L}_{3}^{2)}$ & 50 & 100 & 600 & 5600 & 30600 \\
$\mathrm{~L}_{4}^{2)}$ & 150 & 100 & 600 & 15600 & 90600 \\
$\mathrm{~L}_{5}^{2)}$ & 200 & 100 & 600 & 20600 & 120600 \\
$\mathrm{~L}_{1}^{3)}$ & 8 & 2502 & 5000 & 25016 & 45000 \\
$\mathrm{~L}_{2}^{3)}$ & 16 & 2502 & 5000 & 45032 & 85000 \\
$\mathrm{~L}_{3}^{3)}$ & 50 & 227 & 450 & 11800 & 22950 \\
$\mathrm{~L}_{4}^{3)}$ & 150 & 227 & 450 & 34500 & 67950 \\
$\mathrm{~L}_{5}^{3)}$ & 200 & 227 & 450 & 45850 & 90450 \\
$\left.\mathrm{~L}_{1}^{4}\right)$ & 8 & 976 & 7808 & 15616 & 70272 \\
$\mathrm{~L}_{2}^{4)}$ & 16 & 976 & 7808 & 23424 & 132736 \\
$\mathrm{~L}_{3}^{4)}$ & 50 & 101 & 606 & 5656 & 30906 \\
$\mathrm{~L}_{4}^{4)}$ & 150 & 101 & 606 & 15756 & 91506 \\
$\mathrm{~L}_{5}^{4)}$ & 200 & 101 & 606 & 20806 & 121806 \\
\hline & & & & &
\end{tabular}

for large multicommodity network flow problems, especially for the case of small-sized networks with many commodities.

\section{REFERENCES}

Castro, J. (1995a) An implementation of a primal-dual interior point algorithm with upper bounded variables. Qüestiió, 19, to appear. (written in Catalan)

Castro, J. (1995b) Efficient methods for the solution of multicommodity network flow problems. Ph.D. dissertation, Statistics and Operations Reseach Dept., Universitat Politècnica de Catalunya, Barcelona, Spain. (written in Catalan)

Castro, J. and Nabona, N. (1995) An implementation of linear and nonlinar multicommodity network flows. Accepted for publication in the European Journal of Operational Research.

Choi, I.C. and Goldfarb, D. (1990) Solving multicommodity network flow problems by an interior point method. SIAM Proceedings in Applied Mathematics, 46, 58-69. 
Table $2 \mathrm{CPU}$ seconds of each code for the linear test problems

\begin{tabular}{llllll}
\hline Test & IPM & MINOS & PPRN & MCNF85 & LoQo \\
\hline $\mathrm{L}_{1}^{1)}$ & 7095.4 & 15147.7 & $737.9^{*}$ & 1778.2 & $(d)$ \\
$\mathrm{L}_{2}^{1)}$ & 16737.9 & $(a)$ & 6838.7 & $5651.3^{*}$ & $(d)$ \\
$\mathrm{L}_{3}^{1)}$ & $178.6^{*}$ & 2639.4 & 275.1 & 398.6 & 3402.8 \\
$\mathrm{~L}_{4}^{1)}$ & $1839.9^{*}$ & $(b)$ & 8069.0 & 11319.0 & $(d)$ \\
$\mathrm{L}_{5}^{1)}$ & $1710.0^{*}$ & $(c)$ & 15415.3 & 26479.9 & $(d)$ \\
$\mathrm{L}_{1}^{2)}$ & 12296.3 & $(b)$ & 4962.2 & $4833.0^{*}$ & $(d)$ \\
$\mathrm{L}_{2}^{2)}$ & $(d)$ & $(c)$ & 37470.5 & $34383.0^{*}$ & $(d)$ \\
$\mathrm{L}_{3}^{2)}$ & 287.0 & 1402.8 & $169.2^{*}$ & 466.9 & 5211.1 \\
$\mathrm{~L}_{4}^{2)}$ & $4352.4^{*}$ & 105082.5 & 7605.5 & 15836.2 & $(d)$ \\
$\mathrm{L}_{5}^{2)}$ & $11974.4^{*}$ & $(c)$ & 22218.4 & 81903.5 & $(d)$ \\
$\mathrm{L}_{1}^{3)}$ & 2818.3 & $(b)$ & $1409.2^{*}$ & 2134.1 & $(d)$ \\
$\mathrm{L}_{2}^{3)}$ & 16485.5 & $(c)$ & $14139.8^{*}$ & 14709.9 & $(d)$ \\
$\mathrm{L}_{3}^{3)}$ & $236.3^{*}$ & 3172.8 & 364.9 & 533.9 & 3180.4 \\
$\mathrm{~L}_{4}^{3)}$ & $962.9^{*}$ & $(a)$ & 4480.5 & 6142.8 & $(d)$ \\
$\mathrm{L}_{5}^{3)}$ & $2083.1^{*}$ & $(a)$ & 11736.8 & 19458.0 & $(d)$ \\
$\mathrm{L}_{1}^{4)}$ & 12216.2 & 205836.0 & $3424.7^{*}$ & $(e)$ & $(d)$ \\
$\mathrm{L}_{2}^{4)}$ & $(d)$ & $(c)$ & $40974.1^{*}$ & $(e)$ & $(d)$ \\
$\left.\mathrm{L}_{3}^{4}\right)$ & 114.1 & 918.2 & $39.4^{*}$ & $(e)$ & $(d)$ \\
$\mathrm{L}_{4}^{4)}$ & 584.8 & 15236.6 & $415.8^{*}$ & $(e)$ & $(d)$ \\
$\mathrm{L}_{5}^{4)}$ & $915.2^{*}$ & $(c)$ & 1273.4 & $(e)$ & $(d)$ \\
\hline$(a)$ & & & $(b)$ Error during execution. & $(c)$ Execution too long. \\
$(d)$ & Noo many constraints. & $($ e $)$ Feasibility error. & &
\end{tabular}

DIMACS. (1991) The first DIMACS international algorithm implementation challenge: The bench-mark experiments. Technical Report, DIMACS, New Brunswick, NJ.

Kamath, A.P, Karmarkar, N.K. and Ramakrishnan, K.G. (1993) Computational and Complexity Results for an Interior Point Algorithm on Multicommodity Flow Problems. TR-21/93, Dipartimento di Informatica, Università di Pisa, Italy.

Kennington, J.L. and Helgasson, R.V. (1980) Algorithms for Network Programming. John Wiley \& Sons, Inc., New York, NY.

Kennington, J.L. (1979) A primal partitioning code for solving multicommodity flow problems (version 1). Technical Report 79008. Dept. of Industrial Engineering and Operations Research, Southern Methodist University, Dallas, USA.

Murtagh, B.A. and Saunders, M.A. (1983) MINOS 5.0. User's guide. Dept. of Operations Research, Stanford University, CA, USA.

Vanderbei, R.J. and Carpenter, T.J. (1993) Symmetric indefinite systems for interior point methods. Mathematical Programming, 58, 1-32. 